\title{
ESSENTIAL MAPS AND MANIFOLDS
}

\author{
JEAN-FRANCOIS MERTENS
}

(Communicated by Frederick R. Cohen)

\begin{abstract}
Let $(M, \partial M)$ be a compact $n$-manifold with boundary, orientable over a field $K$ with characteristic $q$. For $f:(Y, \partial Y) \rightarrow(M, \partial M)$, with $Y$ compact, and $(X, \partial X)$ a compact pair, $g: X \rightarrow M$, let $(P, \partial P)=\{(y, x) \in$ $Y \times(X, \partial X) \mid f(y)=g(x)\}$ denote the fibered product, with $p$ as the projection to $(X, \partial X)$. In Čech-cohomology with coefficients $K$, we show that if $\breve{H}^{n}(f)$ is injective then so is $\check{H}^{*}(p)$-and a number of strengthenings, which point to a concept of $q$-essential map from one compact space to another.
\end{abstract}

Let $(M, \partial M)$ be a compact $n$-manifold with boundary, orientable over the ring $R$. For $f:(Y, \partial Y) \rightarrow(M, \partial M)$ and $(X, \partial X)$ a compact pair, $g: X \rightarrow$ $M$, let $(P, \partial P)=\{(y, x) \in Y \times(X, \partial X) \mid f(y)=g(x)\}$ denote the fibered product, with $p$ as the projection to $(X, \partial X)$. Also we fix a coefficient module $G$ over $R$ for homology or cohomology, and any compact space in this paper is assumed Hausdorff.

In [1] we showed that if $(X, \partial X)$ is a $d$-simplex, $g$ one-to-one (this is dispensible, by the methods of the present paper), $M$ connected, and everything is semialgebraic $(M, \partial M, Y, \partial Y, f, g, X$, and $\partial X)$, then letting $G_{1}=$ $f_{*}\left[H_{n}(Y, \partial Y ; G)\right] \subseteq H_{n}(M, \partial M ; G) \sim G$ and $G_{2}=p_{*}\left[H_{d}(P, \partial P ; G)\right] \subseteq$ $H_{d}(X, \partial X ; G) \sim G$, one has $G_{1} \subseteq G_{2}$.

Here we restrict $R$ to be a field and prove an equivalent result (i.e., showing also that cycles can be lifted through $p$ ) without any of the above restrictions. In particular, since we no longer have semialgebraicity, the formulation is rather in terms of a weakly continuous cohomology theory $\breve{H}$, so $Y$ is also assumed compact.

Theorem. If $\check{H}^{n}(f)$ is one-to-one then $\check{H}^{*}(p)$ is also.

Remarks. The result of [1] was a basic tool for [2]; in particular, one needed arbitrary $R$ to show in [2] that it was a specific game theoretic property-the decomposition property - that forced one to use only fields $R$ for defining stable sets. (Those results suggest a look at conjectures of the type: if a class of essential proper maps from locally compact spaces to Euclidean spaces is stable under products (and, say, homotopy invariant) then there is some characteristic $p$ (zero or prime) such that those maps are all essential in the sense of

Received by the editors April 12, 1990.

1991 Mathematics Subject Classification. Primary 55M99; Secondary 55M25.

This work was partially supported by N.S.F. Grant SES8922610. 
Čech-cohomology with compact supports and with coefficients $\mathbb{Z}_{p}$ (with $\mathbb{Z}_{0}=$ $\mathbb{Q})$.) Given that the decomposition property basically limits consideration to fields $R$, the present result is the tool nneded for proving the "small worlds" property mentioned in [2], as shown in a parallel paper [3]. The situation, nevertheless, remains unsatisfactory in that we cannot simultaneously handle (even in a semialgebraic framework) arbitrary coefficient modules and arbitrary compact pairs. Here the point of getting rid of any semialgebraicity restriction is to stress the purely topological nature of such properties.

Lemma 1. For a map $p:(P, \partial P) \rightarrow(X, \partial X)$ of compact pairs, $\check{H}^{*}(p)$ is oneto-one if and only if it is so for some vector space $G^{\prime}$ of positive dimension over the field $R$.

Remark. Therefore, this property depends only on the characteristic $q$ (zero or prime) of the field $R$, since $G$ can always be viewed as a vector space over the prime field $\mathbb{Z}_{q}$. Thus, we can assume $G=R=\mathbb{Z}_{q}$.

Proof of Lemma 1. Use the universal coefficient theorem [5, VI.8.11] (torsion products are zero since $R$ is a field).

Remark. Similarly, orientability of the manifold when the ring $R$ is an algebra over a field $K$ depends only on the characteristic of $K$ and can be expressed purely in terms of Čech-cohomology as the isomorphism of $\breve{H}^{n}(M, \partial M ; K)$ with $\check{H}^{0}(M, \partial M ; K)$.

Lemma 2. Under the assumptions of the theorem (without the map $g$ ), there exists a triangulable, compact, orientable $\bar{n}$-manifold $\bar{M}$, a compact space $\bar{Y}, a$ map $\bar{f}: \bar{Y} \rightarrow \bar{M}$ with $\breve{H}^{\bar{n}}(\bar{f})$ one-to-one, and one-to-one maps $i: M \rightarrow \bar{M}$ and $j: Y \rightarrow \bar{Y}$ such that for every compact pair $(X, \partial X)$ and every map $g: X \rightarrow M$, the fibered product of $i \circ g$ and $\bar{f}$ equals (under $j$ ) that of $g$ and $f$, with the same projection $p$.

Proof of Lemma 2. First observe there is no loss in assuming also $\partial Y$ compact: if it is not, let $\partial Y^{\prime}$ denote its closure, $i:(Y, \partial Y) \subseteq\left(Y, \partial Y^{\prime}\right), f^{\prime}:\left(Y, \partial Y^{\prime}\right) \rightarrow$ $(M, \partial M)$. Then $f=f^{\prime} \circ i$ so $\breve{H}^{n}(f)=\breve{H}^{n}(i) \circ \breve{H}^{n}\left(f^{\prime}\right)$, hence $\check{H}^{n}\left(f^{\prime}\right)$ is also one-to-one; we can work with $\partial Y^{\prime}$ instead of $\partial Y$. By Lemma 1, we can now assume $G$ is the prime field. We first reduce the problem to the case where $\partial M=\partial Y=\varnothing$.

If $\partial M \neq \varnothing$, glue $M$ to a copy of itself along $\partial M$; thus, $\left(M^{+}, \partial M^{+}\right)$and $\left(M^{-}, \partial M^{-}\right)$are two copies of $M$ and $\partial M^{+}$and $\partial M^{-}$are identified. In this way, one obtains $\bar{M}$, which is clearly a compact manifold with subsets $M^{+}, M^{-}$, and $\partial M$. Do the same with $(Y, \partial Y)$, obtaining a compact $\bar{Y}$ that contains $Y^{+}, Y^{-}$, and $\partial Y$. Then $f$ induces naturally a map $\bar{f}: \bar{Y} \rightarrow \bar{M}$. Send $(X, \partial X)$ to the corresponding subsets of $M^{+} \subseteq \bar{M}$. We identify $M$ with $M^{+}$and $Y$ with $Y^{+}$. It follows that the problem will be reduced to the case $\partial M=\partial Y=\varnothing$ if we prove that $\bar{M}$ is orientable and that $\check{H}^{n}(\bar{f})$ is one-to-one. By definition of orientability, and by [5, VI.4.8], it suffices to prove both points on each connected component separately, i.e., we can assume $M$ and $\bar{M}$ connected.

Observe now $\bar{M}$ has a locally flat embedding into some space $\mathbb{R}^{N}$, with $N \geq n-2+\max (8, n)$, i.e., such that every point of $\bar{M}$ has a neighbourhood in $\mathbb{R}^{N}$ that is an $(N-n)$-ball product-bundle with its intersection with $\bar{M}$ as 
base. (For example, choose for each point $x$ an open neighbourhood $U_{x}$ with a homeomorphism $\varphi_{x}$ from $U_{x}$ to the open unit ball $B$ in $\mathbb{R}^{n}$. Let $h(r)=$ $\min \left[1,2(1-r)^{+}\right], \quad V_{x}=\left\{y \in U_{x} \mid\left\|\varphi_{x}(y)\right\|<\frac{1}{2}\right\}, \psi_{x}: \bar{M} \rightarrow \mathbb{R}^{n+1}: \psi_{x}(y)=$ $h\left(\left\|\varphi_{x}(y)\right\|\right)\left(1, \varphi_{x}(y)\right)$ for $y \in U_{x}, \psi_{x}(y)=0$ for $y \notin U_{x} . \psi_{x}$ is clearly continuous and separates points of $U_{x}$ as well as separating each of them from any point not in $U_{x}$. Then let $\left(x_{i}\right)_{i \in I}$ be a finite set such that the $V_{x_{i}}$ cover $\bar{M}$ : the function $\psi=\left(\psi_{x_{i}}\right)_{i \in I}$ is the required embedding, with $N=(\# I)(n+1)$, choosing $\# I \geq 4-\frac{1}{2} n$ to have $N$ sufficiently large. Indeed $\psi$ is clearly injective, and every point $x$ of $\psi(\bar{M})$ has a neighbourhood in $\psi(\bar{M})$ of the form $V_{x_{i}}$ on which the projection $p$ to a subset of $n$ coordinates separates points, so that the $N-n$ others are a continuous function $h$ of those, allowing immediately the construction of the $(N-n)$-ball product-bundle as $W=\left\{\left(x^{\prime}, y^{\prime}\right) \in\right.$ $\left.\mathbb{R}^{n} \times \mathbb{R}^{N-n} \mid d\left(x^{\prime}, p(x)\right) \leq \varepsilon, d\left(y^{\prime}, h\left(x^{\prime}\right)\right) \leq \varepsilon\right\}$, with projection $p$ on the first factor (identified with $W \cap \bar{M}$ ), where $\varepsilon>0$ is choosen sufficiently small so that $W \cap \psi\left(\bar{M} \backslash V_{x_{i}}\right)=\varnothing$.) Therefore, by [6, 4.5], $\bar{M}$ has a normal bundle in $\mathbb{R}^{N}$, i.e., an open neighbourhood $O$ and a retraction $p$ from $O$ to $\bar{M}$ such that $\left(O, \bar{M}, \mathbb{R}^{N-n}, p\right)$ is a fiber bundle [5, II.7] (using also invariance of domain $[5$, IV.8.16] to be sure).

Consider then the open set $U$ in $\bar{M}$ consisting of $M^{+}(=M)$ together with an open collar [5, VI.2] of $\left(M^{-}, \partial M\right)$, with the corresponding retraction $q: U \rightarrow M^{+}$. Let $V=p^{-1}(U)$ and $r: V \rightarrow M: r=q \circ p$. Then $r$ is a retraction from the neighbourhood $V$ of $M$ in $\mathbb{R}^{N}$ to $M$. Embed $Y$ in a cube $C=[0,1]^{I}$, and consider (Tietze) a continuous extension $\tilde{f}$ of $f$ from $C$ to $\mathbb{R}^{N}$. Consider the directed system of sets $C_{\alpha}=Y_{\alpha} \times[0,1]^{I \backslash J_{\alpha}}$ where $J_{\alpha}$ is a finite subset of $I$ and $Y_{\alpha}$ a polyhedron in $[0,1]^{J_{\alpha}}$ such that $Y \subseteq C_{\alpha}$. Since the $C_{\alpha}$ decrease to $Y$, there exists $\alpha_{0}$ such that $\tilde{f}\left(C_{\alpha_{0}}\right) \subseteq U$. Then define $f: C_{\alpha_{0}} \rightarrow M$ as $f=r \circ \tilde{f}_{\mid C_{\alpha_{0}}}$, and henceforth we consider only $\alpha \geq \alpha_{0}$. Given a collaring of $\partial M[5$, VI.6.2] one can construct a homotopy relative to $\partial M$ between the identity on $M$ and a map sending a neighbourhood $V$ of $\partial M$ into $\partial M$. Let $f^{\prime}: C_{\alpha_{0}} \rightarrow M$ be the composition of $f$ with this map; then $f^{\prime}$ is homotopic to $f$ as a map from $(Y, \partial Y)$ into $(M, \partial M)$, so $\check{H}^{n}\left(f^{\prime}\right) \neq 0 ; \bar{f}^{\prime}$ is homotopic to $\bar{f}$ as a map from $\bar{Y}$ to $\bar{M}$, so $\check{H}^{*}\left(\bar{f}^{\prime}\right)=\check{H}^{*}(\bar{f})$. It suffices thus to do the proof for the map $f^{\prime}$; i.e., we can assume that $f: C_{\alpha_{0}} \rightarrow M$ maps a neighbourhood $U$ of $\partial Y$ into $\partial M$. This neighbourhood can, by compactness, be chosen to depend only on finitely many coordinates, say $J_{\alpha}\left(\supseteq J_{\alpha_{0}}\right)$; then let $Y_{\alpha}=Y_{\alpha_{0}} \times[0,1]^{J_{\alpha} \backslash J_{\alpha_{0}}}, C_{\alpha}=C_{\alpha_{0}}$ : a sufficiently fine subdivision of the triangulation of the polyhedron $Y_{\alpha}$ will be such that, for any simplex $\sigma$, letting $\hat{\sigma}=\sigma \times[0,1]^{I \backslash J_{\alpha}}$, if $\hat{\sigma} \cap \partial Y \neq \varnothing$ then $f(\hat{\sigma}) \subseteq \partial M$. Denote by $\partial C_{\alpha}$ the union of all those $\hat{\sigma}$ : we have $\left(C_{\alpha}, \partial C_{\alpha}\right)=\left(K_{\alpha}, \partial K_{\alpha}\right) \times[0,1]^{I \backslash J_{\alpha}}$, where $K_{\alpha}$ and $\partial K_{\alpha}$ are (the space of) a simplicial complex and a (full, by one more subdivision) subcomplex, respectively. Let also $i_{\alpha}:(Y, \partial Y) \subseteq\left(C_{\alpha}, \partial C_{\alpha}\right)$ and $f_{\alpha}:\left(C_{\alpha}, \partial C_{\alpha}\right) \rightarrow(M, \partial M)$. Thus since $\check{H}^{n}(f)=\check{H}^{n}\left(f_{\alpha} \circ i_{\alpha}\right) \neq 0$, we have also $\check{H}^{n}\left(f_{\alpha}\right) \neq 0$. The system $\left(C_{\alpha}, \partial C_{\alpha}\right)$ is directed downwards by inclusion, with intersection $(Y, \partial Y)$; so the $\bar{C}_{\alpha}$ form a projective system with limit $\bar{Y}$ and the maps $\bar{f}_{\alpha}: \bar{C}_{\alpha} \rightarrow \bar{M}$ and $\bar{f}: \bar{Y} \rightarrow \bar{M}$ commute with this system. By the continuity property [5, VI. Example C.2, VI.6.6], it follows that $\breve{H}^{n}(\bar{f})$ will be nonzero if we prove that $\check{H}^{n}\left(\bar{f}_{\alpha}\right)$ is nonzero for each $\alpha$. Thus our problem is 
reduced to the case where $(Y, \partial Y)=(K, \partial K) \times[0,1]^{I}$, with $(K, \partial K)$ a simplicial pair. Now let $h_{t}:(Y, \partial Y) \rightarrow(Y, \partial Y): h_{t}\left(k,\left(x_{i}\right)_{i \in I}\right)=\left(k,\left(t x_{i}\right)_{i \in I}\right)$, $f_{t}=f \circ h_{t}$, and $\pi$ be the projection from $(Y, \partial Y)$ to $(K, \partial K): f_{0}=\phi \circ \pi$ and $f$ are homotopic maps from $(Y, \partial Y)$ to $(M, \partial M)$, and similarly $\bar{f}_{0}$ and $\bar{f}$ are homotopic from $\bar{Y}$ to $\bar{M}$. This thus reduces the problem to the case where furthermore $f=\phi \circ \pi$, where $\phi$ is a map from $(K, \partial K)$ to $(M, \partial M)$. Finally, since $\pi$ is a homotopy equivalence, it suffices to consider the case where $(Y, \partial Y)$ itself is a polyhedral pair. All homology and cohomology theories are now equivalent on $(Y, \partial Y)$ and on $\bar{Y}[5$, IV.8.10, V.5] and on $(M, \partial M)$ and on $\bar{M}$ singular cohomology and Čech-cohomology coincide, respectively ([5, VI.8.8, VI.9.9, VI.1.7] and collaring). Thus we know $H^{n}(f) \neq 0$ and want to prove $H^{n}(\bar{f}) \neq 0$, all in singular cohomology.

By the universal coefficient theorem [5, V.5.3] $H^{n}$ and $H_{n}$ are dual finitedimensional vector spaces; so $H^{n}(f)$ being nonzero is equivalent to $H_{n}(f) \neq$ 0 . Thus, let $c$ be a simplicial $n$-cycle on $(Y, \partial Y)$ that is mapped to a nonzero singular cycle on $(M, \partial M)$ (using [5, IV.6.8]) - thus, to a fundamental class $z$ since $R$ is a field and $(M, \partial M)$ is compact and connected [5, VI.3.8]. Let $c^{+}$and $c^{-}$denote the corresponding chains on $Y^{+}$and on $Y^{-}$; then $\bar{c}=c^{+}-c^{-}$is an $n$-cycle on $\bar{Y}$ with image $\bar{z} \in H_{n}(\bar{M} ; R) . \bar{z}$ is nonzero, e.g., because its image in $H_{n}(\bar{M}, \bar{M}-x ; R)$ equals the nonzero image of $z$ in $H_{n}(M, M-x ; R)$ for $x \in M^{+} \backslash \partial M$ (cf. [5, VI.3.8]). This yields both the orientability of $\bar{M}$ (again [5, VI.3.8]) and that $\check{H}_{n}(\bar{f})$ is nonzero.

Hence we can assume $\partial Y=\partial M=\varnothing$.

Recall now our previous normal bundle $\left(O, M, \mathbb{R}^{N-n}, p\right)$ for $M$ as embedded in $\mathbb{R}^{N}$. In particular, $M$ is a euclidean neighbourhood retract, and so by $[6,1.3]$ the bundle contains a ball-bundle, i.e., there exists a compact pair ("tubular neighbourhood") $(T, \partial T)$, with $T \subseteq O$ and $M \subseteq T \backslash \partial T$, such that the restriction of $p$ to $(T, \partial T)$ is a ball-bundle.

Apply now [5, VI.10.15] to obtain $\theta(1)$ nonzero in $H^{N-n}\left(\mathbb{R}^{N}, \mathbb{R}^{N} \backslash M ; R\right)$. We want to show that the image $U$ of $\theta(1)$ in $(T, \partial T)$ by inclusion and excision (collaring and [5], IV.8.9) is an orientation of the bundle. It suffices to do this separately for each connected component of $M$. For $E \subseteq M$, let $T_{E}=T \cap p^{-1}(E), \partial T_{E}=T_{E} \cap \partial T, \theta_{E}$ is the restriction of $\theta(1)$ to $\left(T_{E}, T_{E} \backslash E\right)$ and $U_{E}$ the restriction of $U$-or of $\theta_{E}$-to $\left(T_{E}, \partial T_{E}\right)$. For $E=\{m\}$, we will write simply $T_{m}$, etc.

Thus assume we had $U_{m}=0$ for some $m$. Since $T_{m}$ is contractible, the connecting homomorphism in the functorial exact cohomology sequences for $\left(T_{m}, \partial T_{m}\right)$ and for $\left(T_{m}, T_{m} \backslash\{m\}\right)$ is an isomorphism; hence the inclusion of the first pair into the second will induce an isomorphism because the inclusion $\partial T_{m} \subseteq T_{m} \backslash\{m\}$ does, being a homotopy equivalence. Thus we have also $\theta_{m}=$ 0 .

Denote then by $\mathscr{V}$ the collection of open sets of $M$ that are homeomorphic to $\mathbb{R}^{n}$ and on which the bundle is a product bundle. For any $V \in \mathscr{V}$ with $m \in V$ we would still have $\theta_{V}=0$ since the inclusion $\left(T_{m}, T_{m} \backslash\{m\}\right) \subseteq$ $\left(T_{V}, T_{V} \backslash V\right)$ is a homotopy equivalence. Hence $\mathscr{V}_{0}=\left\{V \in \mathscr{V} \mid \theta_{V}=0\right\}$ would be nonempty and any $V \in \mathscr{V}_{0}$ disjoint from any $V \in \mathscr{V} \backslash \mathscr{V}_{0}$; by connexity, $\mathscr{V}_{0}=\mathscr{V}$; i.e., $\theta_{V}=0 \forall V \in \mathscr{V}$. Assume now $W$ is an open set in $M$ with $\theta_{W}=0$ and $V \in \mathscr{V}$. Let $\widetilde{W}=W \cup V, S=W \cap V$, and $d=N-n$, and 
consider the exact Mayer-Vietoris sequence [5, V.4.9]:

$$
\begin{aligned}
H^{d-1}\left(T_{S}, T_{S} \backslash S\right) & \stackrel{\delta^{*}}{\rightarrow} H^{d}\left(T_{\widetilde{W}}, T_{\widetilde{W}} \backslash \widetilde{W}\right) \\
& \rightarrow H^{d}\left(T_{W}, T_{W} \backslash W\right) \oplus H^{d}\left(T_{V}, T_{V} \backslash V\right) .
\end{aligned}
$$

Since $S \subseteq V$, it follows that $\left(T_{S}, T_{S} \backslash S\right)$ is a product-bundle, so by Künneth's isomorphism [5, V.6.1] $H^{d-1}\left(T_{S}, T_{S} \backslash S\right)$ is zero. Hence $\theta_{W}$ and $\theta_{V}$ (which are restrictions of $\theta_{\widetilde{W}}$ ) being both zero imply that $\theta_{\widetilde{W}}=0$ also. Therefore, by induction on $k$, we will have $\theta_{W}=0$ for every union $W$ of $k$ elements of $\mathscr{V}$; thus, by compactness, $\theta_{M}=\theta(1)=0$. This contradicts [5, VI.10.15], since $H^{*}(M)$ is not identically zero.

Thus our ball-bundle is orientable.

By [5, V.7.6], the fibered product $\bar{Y}$ of $f$ and $p$ is then an $(N-n)$-ball bundle with the projection $q$ to $Y$, and say $\bar{f}$ as projection to $T$, and $\bar{U}=$ $\bar{f}^{*}(U)$ as orientation. Further our inclusion of $M$ in $T \backslash \partial T$ yields $Y \subseteq$ $\bar{Y} \backslash \partial \bar{Y}$, and $f$ is the restriction of $\bar{f}$ to $Y$. Write also $(\bar{M}, \partial \bar{M})$ for $(T, \partial T)$. Observe first that $(\bar{M}, \partial \bar{M})$, as a tubular neighbourhood, is clearly a compact manifold with boundary, and as embedded in $\mathbb{R}^{N}$, is orientable.

By the Thom isomorphism theorem [5, V.7.10] we have a commuting diagram

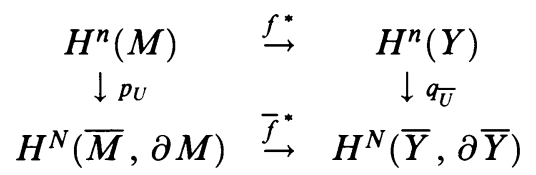

with $p_{U}(\mu)=p^{*}(\mu) \cup U, q_{\bar{U}}(\eta)=q^{*}(\eta) \cup \bar{f}^{*}(U)$, and where the vertical arrows are isomorphisms. Therefore $f^{*}$ being one-to-one would imply $\bar{f}^{*}$ is one-toone. Actually, what we need is a version of this theorem in Čech-cohomology (there are trivial examples that this matters, e.g., projection on $S^{1}$ of the closure of the graph of the curve $\left.\sin \left(\pi^{2} / \theta\right) \quad(0<|\theta| \leq \pi)\right)$ : for such a version, cf. e.g., [2, part II, Appendix IV], and use [5, VI.9.5] and the five lemma for the isomorphism of singular and Čech-cohomology on $M$ and on $(\bar{M}, \partial \bar{M})$.

Thus $\bar{f}:(\bar{Y}, \partial \bar{Y}) \rightarrow(\bar{M}, \partial \bar{M})$ is such that $\check{H}^{N}(\bar{f})$ is one-to-one, that $\bar{f}_{\mid Y}=$ $f$ and that $\bar{f}(\bar{Y} \backslash Y)$ and $\partial \bar{M}$ are disjoint from $M$ (here $Y$ and $M$ denote the original objects). And $(\bar{M}, \partial \bar{M})$ is a compact $N$-manifold with boundary, embedded in $\mathbb{R}^{N}$.

Observe also that connected components of $M$ and $\bar{M}$ correspond to each other. Consider then a cube in $\mathbb{R}^{N}$ containing $\bar{M}$, and subdivide its triangulation until every simplex that intersects $M$ is contained in $\bar{M} \backslash \partial \bar{M}$. Let $K$ be the union of those simplices. Subdivide the triangulation further such that every new simplex that meets $K$ is contained in $\bar{M} \backslash \partial \bar{M}$. Let $\bar{K}$ be the union of those simplices. A further subdivision yields a regular neighbourhood $L$ of $K$ in $\bar{K}$-or in the cube, with further $L \subseteq \bar{M} \backslash \partial \bar{M}$. Hence, by [4, 3.10], $L$ is a compact PL-manifold with boundary $\partial L$; further $M \subseteq L \backslash \partial L$, and the connected components of $L$ correspond bijectively to those of $M$ and of $\bar{M}$, by construction.

Let $\partial \widetilde{M}=(M \backslash L) \cup \partial L$. We want to show that $i^{*}: \check{H}^{N}(\bar{M}, \partial \widetilde{M}) \rightarrow$ $\check{H}^{N}(\bar{M}, \partial \bar{M})$ is one-to-one. It clearly suffices to prove this on each connected component separately. There both spaces are the underlying field $R$ (the first 
by excision), so it suffices to prove $i^{*} \neq 0$. Including a small cube in front and our large cube at the end would otherwise yield by composition that $i^{*}$ is still zero when $L$ is a small ball included in a bigger ball $\bar{M}$, contradicting homotopy invariance. Let $j:(\bar{Y}, \partial \bar{Y}) \subseteq(\bar{Y}, \partial \widetilde{Y})$, with $\partial \widetilde{Y}=\bar{f}^{-1}(\partial \widetilde{M})$, and let $\tilde{f}:(\bar{Y}, \partial \widetilde{Y}) \rightarrow(\bar{M}, \partial \widetilde{M})$ equal $\bar{f}$. Then $\bar{f}^{*} \circ i^{*}=j^{*} \circ \tilde{f}^{*}$, so $\check{H}^{N}(\tilde{f})$ is also one-to-one.

Finally, let $\left(M^{\prime}, \partial M^{\prime}\right)=(L, \partial L) \subseteq(\bar{M}, \partial \widetilde{M})$ and $\left(Y^{\prime}, \partial Y^{\prime}\right)=$ $\tilde{f}^{-1}\left(M^{\prime}, \partial M^{\prime}\right) \subseteq(\bar{Y}, \partial \widetilde{Y})$. By [5, VI.6.5], both inclusions induce isomorphisms in Čech-cohomology; so, with $f^{\prime}:\left(Y^{\prime}, \partial Y^{\prime}\right) \rightarrow\left(M^{\prime}, \partial M^{\prime}\right)$ equal to $\tilde{f}$, we also have $\breve{H}^{N}\left(f^{\prime}\right)$ one-to-one. As before, $f_{\mid Y}^{\prime}=f$ and $f^{\prime}\left(Y^{\prime} \backslash Y\right)$ and $\partial M^{\prime}$ are disjoint from $M$. And now $\left(M^{\prime}, \partial M^{\prime}\right)$ is a compact, orientable $P L$-manifold with boundary.

Finally, repeat the beginning of this proof with those objects to remove the boundaries.

Proof of the theorem. By Lemmas 1 and 2, we can assume that $G=R$ is the prime field (the theorem being trivially true in the zero-dimensional case), and that $M$ is a $P L$-manifold, with $\partial M=\partial Y=0$. Further, by [5, VI.4.8], we can assume $M$ connected. We will first prove the result in the case where $g$ is one-to-one. $X$ can then be viewed as a subspace of $M$ and $(P, \partial P)$ as the inverse image by $f$ of $(X, \partial X)$ in $Y$, with $p$ the restriction of $f$ to this space.

We first reduce this problem to the piecewise-linear case.

Fix a triangulation of $M$ and view $M$ as the space of this simplicial complex, thus as a subcomplex of the simplex $\Delta_{k}$ on the set of vertices of the triangulation. By [5, III Example A.1] $M$ is a neighourhood retract in $\Delta_{k}$, i.e., [5, I Example C.1] there is a neighbourhood $U$ of $M$ in $\Delta_{k}$ and a retraction $r$ from $U$ to $M$. Embed $Y$ in a cube $C=[0,1]^{I}$, and consider a continuous extension $\bar{f}$ of $f$ from $C$ to $\Delta_{k}$. For every finite subset $J$ of $I$, denote by $\pi_{J}$ the projection from $C$ to $[0,1]^{J}$, and let $C_{J}=\pi_{J}^{-1}\left(\pi_{J}(Y)\right)$; since the $C_{J}$ decrease to $Y$, there exists $J_{0}$ such that $\bar{f}\left(C_{J_{0}}\right) \subseteq U$. Define then $f$ on $C_{J_{0}}$ as $f=r \circ \bar{f}$. Now $f: C_{J_{0}} \rightarrow M$, and henceforth, we consider only $J \supseteq J_{0}$. For any $J_{\alpha}\left(\supseteq J_{0}\right)$, let $C_{\alpha}=C_{J_{\alpha}}, f_{\alpha}=f_{\mid C_{\alpha}},\left(P_{\alpha}, \partial P_{\alpha}\right)=f_{\alpha}^{-1}(X, \partial X), \tilde{f}_{\alpha}=f_{\mid\left(P_{\alpha}, \partial P_{\alpha}\right)}$. Since $f: Y \rightarrow M$ factors into an inclusion and $f_{\alpha}$, it follows that $\check{H}^{n}\left(f_{\alpha}\right)$ is one-to-one also. So if the theorem was established for the $C_{\alpha}$ and $f_{\alpha}$, the weak continuity property [5, VI.6.6] will imply the result for $Y$ since $\left(P_{\alpha}, \partial P_{\alpha}\right)$ decreases to $(P, \partial P)$. Thus we can assume $Y=Y_{0} \times[0,1]^{I}$, with $Y_{0}$ finitedimensional. The same argument shows that we can replace $Y_{0}$ by a compact polyhedron containing it, and similarly that we can replace $(X, \partial X)$ by the complex of all simplices intersecting it, for some sufficiently fine subdivision of a triangulation of $M$. We are thus in the case where $(X, \partial X)$ is a pair of full (using one further subdivision) subcomplexes of $M$, and $Y=Y_{0} \times[0,1]^{I}$, where $Y_{0}$ is a finite simplicial complex. If the result were not true, we would have $v \in \check{H}^{*}(X, \partial X), v \neq 0$, and $f^{*}(v)=0$ in $\check{H}^{*}(P, \partial P)$. Use then the weak continuity property as above to find a sufficiently fine subdivision of the triangulation of $M$ such that, denoting by $\left(X_{1}, \partial X_{1}\right)$, the simplicial neighbourhood of (i.e., the union of all simplices of the subdivision intersecting) $(X, \partial X)$, one has $v=i^{*} v_{1}$, for $v_{1} \in \check{H}^{*}\left(X_{1}, \partial X_{1}\right), i:(X, \partial X) \subseteq\left(X_{1}, \partial X_{1}\right)$, 
and such that $f^{*}\left(v_{1}\right)=0$ in $\check{H}^{*}\left(P_{1}, \partial P_{1}\right)$, with $\left(P_{1}, \partial P_{1}\right)=f^{-1}\left(X_{1}, \partial X_{1}\right)$.

Note that $f$, as a continuous map to a compact metric space, depends only on a countable set $I_{0}$ of coordinates in $I$. Since projections on $Y_{0} \times[0,1]^{I_{0}}$ and on $(P, \partial P) \times[0,1]^{I_{0}}$ are homotopy equivalences, we can assume $I$ countable. Then $Y$ is compact metric, and there exists $\varepsilon>0$ such that the image of every ball in $Y$ of radius $\leq \varepsilon$ is contained in some star of the triangulation of $M$. So there exists a finite subset, $I_{0}$ of $I$, and $\delta>0$, such that for any ball $C$ of radius $\leq \delta$ in $Y_{0} \times[0,1]^{I_{0}}, f\left(\pi^{-1}(C)\right)$ is contained in some star of the triangulation of $M$, using $\pi$ for the projection from $Y$ to $Y_{0} \times[0,1]^{I_{0}}$. Since $Y_{0} \times[0,1]^{I_{0}}$ is a polyhedron, we can think of it as $Y_{0}$ itself; and can then subdivide its triangulation such as to have that the star of every vertex has diameter $\leq \delta$; now $Y_{0}$ is a polyhedron, $I$ is countable, and $f\left(\pi^{-1}(C)\right.$ ) is contained in some star of the triangulation of $M$ for every star $C$ in $Y_{0}$ ( $\pi$ projects $Y$ to $Y_{0}$ ). We now use the simplicial approximation theorem. Consider the map $\phi$ mapping every vertex $x$ of $Y_{0}$ to some vertex of $M$ such that $f\left(\operatorname{star}(x) \times[0,1]^{I}\right) \subseteq \operatorname{star}(\phi(x))$, extend $\phi$ by linearity to $Y_{0}$, and define $\bar{f}: Y \rightarrow M$ as $\phi \circ \pi$. $\phi$ is clearly a simplicial map, and for every $y \in Y$ the simplex spanned by $f(y)$ contains $\bar{f}(y)$.

So $\left(P_{2}, \partial P_{2}\right)=\bar{f}^{-1}(X, \partial X)$ is a pair of subcomplexes of $Y_{0}\left(\times[0,1]^{I}\right)$ with $\left(P_{2}, \partial P_{2}\right) \subseteq\left(P_{1}, \partial P_{1}\right)$. Thus the linear homotopy connecting $f$ and $\bar{f}$ is a homotopy both for maps from $Y$ to $M$ and for maps from $\left(P_{2}, \partial P_{2}\right)$ to $\left(X_{1}, \partial X_{1}\right)$. Hence our assumption on $f$ still applies to $\bar{f}$, and the following diagram is homotopy-commutative:

$$
\begin{array}{ccc}
\left(P_{2}, \partial P_{2}\right) & \stackrel{j}{\rightarrow} & \left(P_{1}, \partial P_{1}\right) \\
\downarrow \bar{f} & & \downarrow f \\
(X, \partial X) & \stackrel{i}{\rightarrow} & \left(X_{1}, \partial X_{1}\right)
\end{array}
$$

Then $f^{*}\left(v_{1}\right)=0$ implies $0=\left(j^{*} \circ f^{*}\right)\left(v_{1}\right)=\bar{f}^{*}\left(i^{*}\left(v_{1}\right)\right)=\bar{f}^{*}(v)$; the result is also not true for the map $\bar{f}=\phi \circ \pi$.

Since $\pi$ is a homotopy equivalence, it follows finally that the result is also false for the simplicial map $\phi$ from the polyhedron $Y_{0}$ to $M$ : it suffices to prove the theorem when $(X, \partial X)$ is a pair of (full) subcomplexes of $M, Y$ (the space of) a finite simplicial complex, and $f$ a simplicial map. $(P, \partial P)$ is then also a polyhedral pair, so that now all homology and cohomology theories are equivalent.

Next we show how to reduce the problem to the case $\partial X=\varnothing$.

Since we are in the simplicial case and coefficients belong to a field, the universal coefficient theorems yield that $H_{q}$ and $H^{q}$ are dual finite-dimensional vector spaces, so $f^{*}$ being one-to-one is equivalent to $f_{*}$ being onto. We have to show that every cycle on $(X, \partial X)$ can be lifted to a cycle on $(P, \partial P)$. Let $S^{1}=[-1,1]$, where 1 and -1 are identified, and let $Y^{\prime}=Y \times S^{1}$, $M^{\prime}=M \times S^{1}$, and $f^{\prime}=f \times \mathrm{id}_{S^{1}}$. For $x \in X$ let $x^{+}=(x, d(x, \partial X)) \in M^{\prime}$ and $x^{-}=(x,-d(x, \partial X))$, using for $d$ a piecewise linear distance of diameter $<1 . X^{+}$and $X^{-}$are the images of $X$ in $M^{\prime}$ under those maps and note $\partial X^{+}=\partial X^{-}=\partial X$. Also, by the Künneth formula, our assumptions are still valid for $Y^{\prime}, M^{\prime}$, and $f^{\prime}$. Then if $c$ is a cycle on $(X, \partial X)$, it can be viewed as a chain on $X^{+}$; subtracting the corresponding chain on $X^{-}$yields a cycle $c^{\prime}$ on $X^{\prime}=X^{+} \cup X^{-}$; let $\tilde{c}^{\prime}$ be a cycle in $P^{\prime}$ mapped to $c^{\prime}$. If $\tilde{c}$ denotes the 
chain $\tilde{c}^{\prime}$ where the coefficients of all simplices that are not sent to $X^{+}$are set to zero, then $\tilde{c}$ is a cycle on $\left(P^{+}, \partial P\right)$ mapped to the cycle $c$ on $\left(X^{+}, \partial X\right)$. The homeomorphism setting the $S^{1}$-coordinate to zero yields the conclusion for the original sets $(X, \partial X)$ and $(P, \partial P)$.

Observe finally that it suffices to prove the theorem in the case where $X$ is connected; otherwise, $X$ splits into finitely many connected components whose inverse images in $Y$ are separated, so that all homology and cohomology groups decompose into the corresponding direct sums [5, IV.4.5, V.4.10]: it suffices to have the result on each connected component separately.

Consider now $v \in H^{d}(X), v \neq 0$. By the above-mentioned duality between homology and cohomology, there exists $z \in H_{d}(X)$ with $v \cap z \neq 0$ [5, V.6.19]. Now follow the proof of [5, VI.10.15]: by [5, VI.9.2], Lemma VI.10.14 still applies; use VI.9.8, VI.9.9 and VI.9.2 to find $V$ and $v^{\prime}$, and the above-mentioned $z$ instead of using VI.3.12. One thus obtains $u \in H^{n-d}(M, M \backslash X)$ such that $u \cup v \in H^{n}(M, M \backslash X)$ is nonzero.

By [5, VI.1.11, V.6.8, and the definition of the cup product before VI.10.15], one obtains the commutative diagram

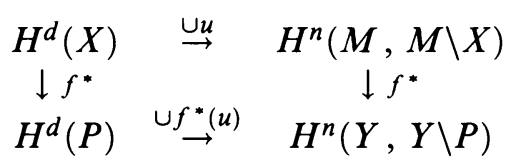

Since $u \cup v \neq 0$, to prove $f^{*}(v) \neq 0$, it suffices to prove that the right-hand map $f^{*}$ is one-to-one.

The functoriality of the cohomology sequence [5, V.4.13] yields the commutative diagram

$$
\begin{array}{ccc}
H^{n}(M, M \backslash X) & \stackrel{i^{*}}{\rightarrow} & H^{n}(M) \\
\downarrow f^{*} & & \downarrow f^{*} \\
H^{n}(Y, Y \backslash P) & \rightarrow & H^{n}(Y)
\end{array}
$$

Hence, the right-hand map $f^{*}$ being one-to-one by assumption, the left-hand one will also be-thus finishing the proof-as soon as we show that $i^{*}$ : $H^{n}(M, M \backslash X) \rightarrow H^{n}(M)$ is one-to-one. By the universal coefficient theorem [5, V.5.3, $R$ is a field], $i^{*}$ is the transpose of $i_{*}: H_{n}(M) \rightarrow H_{n}(M, M-X)$, so it suffices to prove the latter is onto. Because singular homology has compact supports [5, IV.4.6], applying the five lemma to the exact homology sequence yields that $H_{n}(M, M-X)$ is the direct limit of $H_{n}(M, M-V)$ when $V$ varies over the open neighbourhoods of $X$. Thus by taking a sufficiently fine subdivision of the triangulation of $M$, it suffices to show that $i_{*}: H_{n}(M) \rightarrow H_{n}(M, M-\stackrel{\circ}{V})$ is onto where $\stackrel{\circ}{V}$ is the union of the stars of all vertices of $X$. Denote by $V$ the closure of $\stackrel{\circ}{V}$ and let $\partial V=V-\stackrel{\circ}{V}$. By excision, $H_{n}(M, M-\stackrel{\circ}{V})=H_{n}(V, \partial V)$ and $(V, \partial V)$ is an $n$-dimensional pseudomanifold with boundary [5, III. Example $\mathrm{C}]$ because $M$ is an $n$-manifold and the subcomplex $X$ is connected. The result is now obvious in simplicial homology theory: there are no boundaries in dimension $n$, the space of $n$-cycles on $(V, \partial V)$ is (at most) onedimensional [5, IV. Example E.1], and for the same reason a nonzero $n$-cycle in $M$ (which exists, by orientability) assigns a nonzero coefficient to each simplex, hence its restriction to $(V, \partial V)$ is a nonzero $n$-cycle.

This proves thus the result when $g$ is one-to-one. 
Consider now the general case, but assume first $X$ is finite-dimensional; i.e., $X$ can be embedded in $\mathbb{R}^{k}$. Denote by $h$ such an embedding in $S^{k}$, and let $(\tilde{Y}, \partial \widetilde{Y})=(Y, \partial Y) \times S^{k},(\widetilde{M}, \partial \widetilde{M})=(M, \partial M) \times S^{k}, \tilde{f}=f \times \mathbb{1}_{S^{k}}$, and $\tilde{g}=$ $(g, h): X \rightarrow \widetilde{M}$. Our previous result can be applied to $(\widetilde{M}, \partial \widetilde{M}, \widetilde{Y}, \partial \widetilde{Y}, \tilde{f}$, $X, \partial X, \tilde{g})$ so that $\tilde{p}:(\widetilde{P}, \partial \widetilde{P}) \rightarrow(X, \partial X)$ is one-to-one in cohomology. But $(\widetilde{P}, \partial \widetilde{P})$ projects (homeomorphically) to $(P, \partial P)$, say by a map $q$ (inverse given by $h)$, and $\tilde{p}=p \circ q$, so $\check{H}^{*}(\tilde{p})=\check{H}^{*}(q) \circ \check{H}^{*}(p): \check{H}^{*}(p)$ is also one-toone.

Assume now $(X, \partial X)=\left(X_{0}, \partial X_{0}\right) \times[0,1]^{I}$, with $X_{0}$ finite-dimensional, $g=g_{0} \circ \pi, \pi$ denoting the projection of $(X, \partial X)$ onto $\left(X_{0}, \partial X_{0}\right)$. Then also, $(P, \partial P)=\left(P_{0}, \partial P_{0}\right) \times[0,1]^{I}$ and $p=p_{0} \times i d_{[0,1]^{I}}$. The previous case yields that $\check{H}^{*}\left(p_{0}\right)$ is one-to-one: hence (e.g., by the functoriality of Künneth's formula for Čech-cohomology), $\check{H}^{*}(p)$ is one-to-one also.

In general, view (triangulation) as before $M$ as a subcomplex of $\Delta_{k}$ and $X$ as a subset of the cube $[0,1]^{I}$, with as first $(k+1)$ coordinates the compositions of $g$ with the coordinate mappings of $\Delta_{k}$ denoted by $I_{0}$. Denote by $\pi_{0}$ the projection on $[0,1]^{I_{0}}$, let $X_{0}=\pi_{0}(X): g$ can be viewed as a (continuous) map, say $g_{0}$, from $X_{0}$ to $M$, so we can extend $g$ to $\pi_{0}^{-1}\left(X_{0}\right)$ by $g=g_{0} \circ \pi_{0}$. For any finite subset $I_{\alpha}$ of $I$ containing $I_{0}$, let $\left(X_{\alpha}, \partial X_{\alpha}\right)=\left[\pi_{\alpha}(X, \partial X)\right] \times$ $[0,1]^{I \backslash I_{\alpha}}:$ the $\left(X_{\alpha}, \partial X_{\alpha}\right)$ decrease to $(X, \partial X)$, the corresponding $\left(P_{\alpha}, \partial P_{\alpha}\right)$ to $(P, \partial P)$, and the maps $p_{\alpha}$ that all commute with those inverse systems satisfy for all $\alpha$ that $\check{H}^{*}\left(p_{\alpha}\right)$ is one-to-one, by the previous case. Thus, by the weak continuity property [5, VI.6.6], it follows that also in the limit $\check{H}^{*}(p)$ is one-to-one. This finishes the proof.

We obtain the following sharpening (similar to the previously mentioned application) only under an additional assumption of metrisability, which "should not" be there.

Proposition 1. If in addition $X$ is metrisable there exists a closed subset $\widetilde{P}$ of $P$ such that $\check{H}^{0}(\tilde{p}): \check{H}^{0}(X) \rightarrow \check{H}^{0}(\widetilde{P})$ is an isomorphism and such that for the fibered product $\bar{p}$ of $\tilde{p}$ with any map $\tilde{g}: \tilde{X} \rightarrow X$, where $(\tilde{X}, \partial \tilde{X})$ is a compact pair, one has that $\check{H}^{*}(\bar{p})$ is one-to-one.

Proof. We first assume $(X, \partial X)$ an orientable $d$-manifold with boundary. Increasing the dimensions of $Y$ and $M$, as at the end of the proof of the theorem, we can assume $g$ is one-to-one, hence the inclusion $X \subseteq M$. For each of the finitely many connected components $\left(X_{\alpha}, \partial X_{\alpha}\right)$ of $(X, \partial X)$, let $\left(Y_{\alpha}, \partial Y_{\alpha}\right)=f^{-1}\left(X_{\alpha}, \partial X_{\alpha}\right)$, and let $f_{\alpha}$ be the restriction of $f$ to $\left(Y_{\alpha}, \partial Y_{\alpha}\right)$ (and $\left.\left(X_{\alpha}, \partial X_{\alpha}\right)\right)$. By the above theorem, we know $\check{H}^{d}\left(f_{\alpha}\right)$ is one-to-one. Let $\pi=\left\{O_{\beta} \mid \beta \in B\right\}$ be an open partition of $Y_{\alpha}$ and $\partial O_{\beta}=O_{\beta} \cap \partial Y_{\alpha}$. Then $\check{H}^{d}\left(Y_{\alpha}, \partial Y_{\alpha}\right)=\Pi_{\beta} \check{H}^{d}\left(O_{\beta}, \partial O_{\beta}\right)$, by [5, VI.4.8] to be extended by exactness and five lemma to pairs. Hence, there exists $O_{\pi} \in \pi$ such that $\check{H}^{d}\left(f_{\alpha}^{\pi}\right)$ is oneto-one, letting $f_{\alpha}^{\pi}$ be the restriction of $f^{\alpha}$ to $\left(O_{\pi}, \partial O_{\pi}\right)$. Denote by $\mathscr{U}$ an ultrafilter over the partitions $\pi$, and let $V=\lim _{\mathscr{U}} O_{\pi}$. Clearly $V$ is compact and connected. Further, let $\forall u \in \mathscr{U}, K_{u}=\operatorname{cl}\left(\bigcup_{\pi \in u} O_{\pi}\right)$, with $\partial K_{u}=K_{u} \cap \partial Y_{\alpha}$, $\partial V=V \cap \partial Y_{\alpha}$. Then $\check{H}^{d}\left(X_{\alpha}, \partial X_{\alpha}\right) \rightarrow \check{H}^{d}\left(K_{u}, \partial K_{u}\right)$ is injective for all $u \in \mathscr{U}$ since its composition with $\check{H}^{d}\left(K_{u}, \partial K_{u}\right) \rightarrow \check{H}^{d}\left(O_{\pi}, \partial O_{\pi}\right)$ is so for $\pi \in u$. Since $(V, \partial V)=\bigcap_{u \in \mathscr{U}}\left(K_{u}, \partial K_{u}\right)$, it follows then from [5, VI.6.6] 
that $\check{H}^{d}\left(X_{\alpha}, \partial X_{\alpha}\right) \rightarrow \check{H}^{d}(V, \partial V)$ is one-to-one. Now select such a set $V$ (or $\left.V_{\alpha}\right)$ for each $X_{\alpha}$, and denote by $\widetilde{P}$ their union: then $\breve{H}^{k}(\tilde{p}): \breve{H}^{k}(X, \partial X) \rightarrow$ $\breve{H}^{k}(\widetilde{P}, \partial \widetilde{P})$ is one-to-one for $k=d$, and thus is so in all dimensions by the above theorem, and $\check{H}^{0}(\tilde{p}): \check{H}^{0}(X) \rightarrow \check{H}^{0}(\widetilde{P})$ is an isomorphism.

Now consider the general case.

Embed $X$ in the cube $[0,1]^{\mathbb{N}}$, as at the end of the proof of the theorem, with $g=g_{0} \circ \pi_{0}$, where $\pi_{0}$ is the projection on $[0,1]^{k}, X_{0}=\pi_{0}(X)$, and $g_{0}: X_{0} \rightarrow M$. As in the beginning of the proof of Lemma 2, $g_{0}$ can then be extended as a continuous map-still $g_{0}$-from a neighbourhood $V_{0}$ of $X_{0}$ to $M$. Construct now inductively a decreasing basis of neighbourhoods $W_{n}$ of $X$ in $[0,1]^{\mathbb{N}}$, with $W_{n}=U_{n} \times[0,1]^{\mathbb{N} \backslash I_{n}}, I_{n}=\{1, \ldots, k+n\},\left(U_{n}, \partial U_{n}\right)$ a manifold with boundary, and a pair of subcomplexes of a subdivision of $[0,1]^{I_{n}}$. Note first that using the regular neighbourhood theorem [4, Proposition 3.10], every compact subset of a compact, triangulated manifold with boundary has a basis of neighbourhoods that are compact manifolds with boundary and subcomplex pairs of some subdivision of the triangulation (find first an appropriate neighbourhood that is a subcomplex in some subdivision, next use the cited theorem). Thus let $X_{n}=\pi_{n}(X)$, with $\pi_{n}$ the projection on $[0,1]^{I_{n}}$ and obtain so inductively $U_{n}$ as a neighbourhood of $X_{n}$ contained in $V_{n}$ with $d\left(u, X_{n}\right) \leq \frac{1}{n}$ for all $u$ in $U_{n}$, denoting by $d$ the maximum distance, and let $V_{n+1}=U_{n} \times[0,1]^{I_{n+1} \backslash I_{n}}$.

Apply then the previous case inductively, to obtain subsets $P_{n}$ of the fibered product of $f$ and $g_{n}$ in $U_{n} \times Y$, with $g_{n}: U_{n} \rightarrow M$ the composition of the projection and $g_{0}$, such that, for the corresponding projection $p_{n}: P_{n} \rightarrow U_{n}$ one has $\check{H}^{*}\left(p_{n}\right): \breve{H}^{*}\left(U_{n}, \partial U_{n}\right) \rightarrow \check{H}^{*}\left(P_{n}, \partial P_{n}\right)$ is one-to-one and $\check{H}^{0}\left(p_{n}\right): \check{H}^{0}\left(U_{n}\right)$ $\rightarrow \check{H}^{0}\left(P_{n}\right)$ is isomorphic (to construct $P_{n+1}$, use $p_{n}$ for $f$ and the projection from $U_{n+1}$ to $U_{n}$ for $g$ ). Let $\widetilde{P}_{n}=P_{n} \times[0,1]^{\mathbb{N} \backslash I_{n}}, \tilde{p}_{n}=p_{n} \times 1: \widetilde{P}_{n} \rightarrow W_{n}$ : by homotopy equivalence, those have still the same properties. And since $\widetilde{P}_{n}$ and $W_{n}$ decrease to $\widetilde{P}_{\infty}$ and $X$, we have indeed from [5, VI.6.6] that $\breve{H}^{0}\left(\tilde{p}_{\infty}\right): \breve{H}^{0}(X) \rightarrow \breve{H}^{0}\left(\widetilde{P}_{\infty}\right)$ is an isomorphism. For a compact pair $(\widetilde{X}, \partial \widetilde{X})$, with $\tilde{g}: \widetilde{X} \rightarrow X$, apply the previous theorem with each $p_{n}$ as $f$ and go similarly to the limit.

Remark. One way to reformulate the above is to define the following concept of "homologically onto in characteristic $p$ ":

Definition. A map $f: X \rightarrow Y$ (both spaces compact) is $p$-essential iff for every compact pair $(Z, \partial Z)$ and any map $g: Z \rightarrow Y, \breve{H}^{*}(q)$ is one-to-one, where $q$ is the projection on $(Z, \partial Z)$ of the fibered product $Q$ of $f$ and $g$, with $\partial Q=q^{-1}(\partial Z)$.

(Ground ring is a field of characteristic $p$.)

Then we obtain the following properties, either straight from the definition or from the theorem (the first of them shows that we indeed generalise exactly the usual concept where $Y$ is a manifold).

(a) If $f:(Y, \partial Y) \rightarrow(M, \partial M)$ is as in the theorem, then $f: Y \rightarrow M$ is $p$-essential.

(b) If $f: X \rightarrow Y$ is $p$-essential and $\partial Y \subseteq Y$, with $\partial X=f^{-1}(\partial Y)$, then $\check{H}^{*}(f): \check{H}^{*}(Y, \partial Y) \rightarrow \check{H}^{*}(X, \partial X)$ is one-to-one. 
(c) If $f: X \rightarrow Y$ is $p$-essential and $g: Z \rightarrow Y$, then the projection from the fibered product of $f$ and $g$ to $Z$ is $p$-essential.

(d) A composition of $p$-essential maps is still so.

(e) $f \circ g$ p-essential implies $f \quad p$-essential.

In addition, the proposition suggests the conjecture that if $f: X \rightarrow Y$ is $p$-essential, there exists a compact $X_{0} \subseteq X$, with $f_{0}: X_{0} \rightarrow Y$ still $p$-essential, and $\breve{H}^{0}\left(f_{0}\right)$ isomorphic. The above proof establishes this conjecture only when

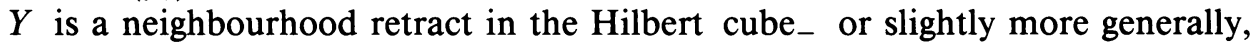
under this assumption, any projection as $\operatorname{sub}(\mathrm{c})$ from the fibered product to $Z$ (metrisable) will have this property.

Remark. The proposition is not fully satisfactory since, for instance in the previously mentioned application, one knows $X \backslash \partial X$ is connected and one needs a subset $\widetilde{P}$ with $\widetilde{P} \backslash \partial \widetilde{P}$ connected. (There, connexity is equivalent to variants like: every compact subset has a compact connected neighourhood.) This we try to improve in the following. We first prove essentially another version of the above conjecture (Proposition 2), and Proposition 3 will give the results in the form that is actually needed.

Proposition 2. Assume $f:(Y, \partial Y) \rightarrow(M, \partial M)$ is as in the theorem and that the $X_{n}$ are compact metric spaces, with $g_{n}: X_{n} \rightarrow X_{n-1}\left(\right.$ and $\left.X_{0}=M\right)$, with $X_{n}$ connected for $n \geq 1$. Let $h_{n}=g_{n} \circ h_{n-1}, h_{0}=\mathbb{1}_{M}$. Denote by $Z_{n}$ the fibered product of $f$ and $h_{n}$, and by $p_{n}$ the projection from $Z_{n}$ to $X_{n}$.

Then there exist compact connected subsets $P_{n}$ of $Z_{n}$, with $\left(g_{n} \times \mathbb{1}_{Y}\right)\left(P_{n}\right) \subseteq$ $P_{n-1}$, such that, denoting by $\bar{p}_{n}$ the restriction of $p_{n}$ to $P_{n}$, for any compact pair $(X, \partial X)$, any $n$, and any map $g: X \rightarrow X_{n}$, the projection $q_{n}$ from the fibered product $\left(Q_{n}, \partial Q_{n}\right)$ of $\bar{p}_{n}$ and $g$ to $(X, \partial X)$ is injective in Cech-cohomology.

Further, the choice of $P_{n}$ can be made completely independently of the $X_{i}$ and $g_{i}$ with $i>n$.

Proof. As in the proof of Proposition 1, construct first inductively an embedding of $X_{n}$ in $[0,1]^{\mathbb{N}}$ and a decreasing basis of neighbourhoods $V_{n}^{i}=U_{n}^{i} \times[0,1]^{J_{n}^{i}}$ of $X_{n}$ in $[0,1]^{\mathbb{N}}$ such that each $\left(U_{n}^{i}, \partial U_{n}^{i}\right)$ is a connected manifold with boundary, such that $g_{n}$ can be viewed as a continuous map from $V_{n}^{i}$ to $V_{n-1}^{i}$ for each $i$, and such that $\phi_{n}^{i}=\operatorname{Proj}_{U_{n-1}^{i}} \circ g_{n}$ is defined on $U_{n}^{i}$ (with $U_{0}^{i}=M$ ).

[For this, construct first of all $U_{1}^{i}$, as in Proposition 1. Once all $U_{n-1}^{i}$ are constructed (viewed as the spaces of simplicial complexes), rank first the compositions of $g_{n}$ with the coordinate mappings of $U_{n-1}^{1}$, then with those of $U_{n-1}^{2}$, etc. Denote by $\phi_{i}$ the corresponding sequence. Intersperse the $\phi_{i}$ with whatever sequence of continuous maps from $X_{n}$ to $[0,1]$ is needed to separate points of $X_{n}$, and use the resulting sequence to define the embedding of $X_{n}$ in $[0,1]^{\mathbb{N}}$. Then extend $g_{n}$ as a continuous map from $[0,1]^{\mathbb{N}}$ (as containing $\left.X_{n}\right)$ to $[0,1]^{\mathbb{N}}$ (as containing $\left.X_{n-1}\right)$, and let $W_{n}^{i}=g_{n}^{-1}\left(V_{n-1}^{i}\right)$. Then select $J_{n}^{i}=\left\{j_{n}^{i}, j_{n}^{i}+1, \ldots\right\}$, and $U_{n}^{i}$ an appropriate (as in Proposition 1) neighbourhood of the projection of $X_{n}$ on $[0,1]^{\mathbb{N} \backslash J_{n}^{i}}$ such that in addition (by taking $j_{n}^{i}$ sufficiently large) one has $V_{n}^{i} \subseteq W_{n}^{i}$ and that the compositions of $g_{n}$ with the coordinate mappings of all $U_{n-1}^{k}$ for $k \leq i$ belong to $\mathbb{N} \backslash J_{n}^{i}$.]

Then select for each $i$, by induction over $n$, using each time, e.g., Proposition 1, a compact connected subset $P_{n}^{i}\left(\subseteq U_{n}^{i} \times Y\right)$ of the fibered product of 
$p_{n-1}^{i}$ and $\phi_{n}^{i}$, with $p_{n}^{i}: P_{n}^{i} \rightarrow U_{n}^{i}$ the corresponding projection $\left(P_{0}^{i}\right.$, the fibered product of $f$ and $\mathbb{1}_{M}$, is not necessarily connected) such that $\check{H}^{*}\left(p_{n}^{i}\right)$ is oneto-one.

Let $Q_{n}^{i}=P_{n}^{i} \times[0,1]_{n}^{J_{n}^{i}} \subseteq V_{n}^{i} \times Y$, with $q_{n}^{i}: Q_{n}^{i} \rightarrow V_{n}^{i}$ the projection: $\check{H}^{*}\left(q_{n}^{i}\right)$ is also one-to-one and $Q_{n}^{i}$ compact connected. Further $g_{n}$ maps $Q_{n}^{i}$ into $Q_{n-1}^{i}$. Extract now a subsequence of $i$ 's such that, for each $n$, the $Q_{n}^{i}$ converge, say to $P_{n}$, in the Hausdorff topology on compact subsets. Then $P_{n}$ is a compact, connected subset of $X_{n} \times Y$, with the projection $\bar{p}_{n}: P_{n} \rightarrow X_{n}$, such that $\check{H}^{*}\left(\bar{p}_{n}\right)$ is one-to-one, and with $g_{n} \times \mathbb{1}_{Y}$ mapping $P_{n}$ into $P_{n-1}$. (In particular, $P_{n} \subseteq Z_{n}$, and is independent of the $X_{i}$ and the $g_{i}$ with $i>n$.)

Finally, given a compact pair $(X, \partial X)$ and a map $g: X \rightarrow X_{n}$, apply the theorem to the fibered product of $q_{n}^{i}: Q_{n}^{i} \rightarrow V_{n}^{i}$ and $g$, and go as above to the limit over $i$ using weak continuity.

Proposition 3. Assume $f:(Y, \partial Y) \rightarrow(M, \partial M)$ is as in the theorem and that $(X, \partial X)$ is a compact metric pair, where each compact subset of $X \backslash \partial X$ is contained in a compact connected subset. If $g: X \rightarrow M$ there exists a compact subset $P$ of the fibered product of $f$ and $g$ such that, with $p$ as the projection to $X$ and $\partial P=p^{-1}(\partial X)$, one has that

- $P$ is the closure of $P \backslash \partial P$ and $P \backslash \partial P$ is connected;

- for every compact pair $(Z, \partial Z)$, with $h: Z \rightarrow X$ and $h^{-1}(\partial X) \subseteq$ $\partial Z$, the projection $q$ from the fibered product $(Q, \partial Q)$ of $p$ and $h$ to $(Z, \partial Z)$ is cohomologically one-to-one.

Proof. Let $K_{n}$ be a sequence of compact, connected subsets of $X \backslash \partial X$ with $K_{n} \subseteq \operatorname{int}\left(K_{n+1}\right)$ and $\bigcup_{n} K_{n}=X \backslash \partial X$. Let $\partial K_{n}=K_{n} \backslash \operatorname{Int}\left(K_{n}\right)$.

Use Proposition 2, with the inclusion maps $g_{n}: K_{n} \subseteq K_{n+1}$, to construct for each $n$

- first $P_{n, n} \subseteq\left\{(y, x) \in Y \times K_{n} \mid f(y)=g(x)\right\}$ compact connected;

- then, $\forall i<n, P_{n, i} \subseteq P_{n, i+1} \cap\left(Y \times K_{i}\right)$ compact connected such that the projections $p_{n, i}: P_{n, i} \rightarrow K$ are essential in the sense of Proposition 2.

In particular, letting $(X, \partial X)=\left(K_{i}, \partial K_{i}\right)$ with the identity map for $g$, we obtain that $q_{n, i}^{*}$ is one-to-one, with $q_{n, i}:\left(P_{n, i}, \partial P_{n, i}\right) \rightarrow\left(K_{i}, \partial K_{i}\right)$ being the projection.

Go to the limit $\left[\left(P_{i}, \partial P_{i}\right), q_{i}\right]$ along a subsequence of indices $n$ along which, for all $i, P_{n, i}$ and $\partial P_{n, i}$ converge in the Hausdorff topology. Then the $P_{i}$ are compact connected, with $P_{i} \subseteq P_{i+1} \cap\left\{(y, x) \in Y \times K_{i} \mid f(y)=g(x)\right\}$, $q_{i}\left(\partial P_{i}\right) \subseteq \partial K_{i}$, and $q_{i}^{*}$ one-to-one.

Let $P$ be the closure of $\bigcup_{i} P_{i}$ in $Y \times X$, with projection $p$ on $X$, and $\partial P=p^{-1}(\partial X)$. Clearly $P$ is a compact subset of the fibered product of $f$ and $g$; since $\left(\bigcup_{i} P_{i}\right) \cap \partial P=\varnothing$, it follows also that $P$ is the closure of $P \backslash \partial P$ and that $\bigcup_{i} P_{i}$ is dense in $P \backslash \partial P$, and hence that $P \backslash \partial P$ is connected since each $P_{i}$ is so and $P_{i} \subseteq P_{i+1}$.

Let $\partial X_{n}=X \backslash \operatorname{int}\left(K_{n}\right), \partial P^{n}=p^{-1}\left(\partial X_{n}\right)$, and $p^{n}:\left(P, \partial P^{n}\right) \rightarrow\left(X, \partial X_{n}\right)$. Then $\bigcap_{n} \partial X_{n}=\partial X, \bigcap_{n} \partial P^{n}=\partial P$, and, because of the excision isomorphism $\left(K_{n}, \partial K_{n}\right) \subseteq\left(X, \partial X_{n}\right)$ and the inclusion $\left(P_{n}, \partial P_{n}\right) \subseteq\left(P, \partial P^{n}\right)$, the injectivity of $p_{n}^{*}$ follows from that of $q_{n}^{*}$.

Similarly, let $\partial Z_{n}=\partial Z \cup h^{-1}\left(\partial X_{n}\right), \partial Q_{n}=q^{-1}\left(\partial Z_{n}\right)$. Then the $\partial Z_{n}$ 
and the $\partial Q_{n}$ decrease to $\partial Z$ and $\partial Q$. Also, by Proposition 2 and excision, let $Q_{k, n}$ denote the fibered product of $p_{k, n}: P_{k, n} \rightarrow X$ and of $h: Z \rightarrow X$, with $q_{k, n}$ as projection to $X$, and $\partial Q_{k, n}=q_{k, n}^{-1}\left(\partial Z_{n}\right)$. Then $\check{H}^{*}\left(q_{k, n}\right): \check{H}^{*}\left(Z, \partial Z_{n}\right)$ $\rightarrow \check{H}^{*}\left(Q_{k, n}, \partial Q_{k, n}\right)$ is one-to-one. The Hausdorff convergence of $P_{k, n}$ to $P_{n}$, together with weak continuity, and the inclusion of $\lim _{k}\left(Q_{k, n}, \partial Q_{k, n}\right)$ in $\left(Q, \partial Q_{n}\right)$, therefore, yield the injectivity of $\check{H}^{*}\left(q_{n}\right): \check{H}^{*}\left(Z, \partial Z_{n}\right)$ $\rightarrow \check{H}^{*}\left(Q, \partial Q_{n}\right)$, and hence the result by a last use of weak continuity.

\section{ACKNOWLEDGMENT}

The author is indebted to an anonymous referee for a very helpful suggestion on how to remove a triangulability assumption on $M$.

\section{REFERENCES}

1. J. F. Mertens, Localisation of the degree on lower dimensional sets, CORE DP 8605, Université Catholique de Louvain, Louvain-la-Neuve, 1986, Math. Oper. Res. (to appear).

2. __ Stable equilibria-a reformulation, CORE DP 8838 Université Catholique de Louvain, Louvain-la-Neuve, 1988, Part I: Definition and basic properties, Math. Oper. Res. 14 (1989), 575-625; Part II: Discussion of the definition and further results, Math. Oper. Res. (to appear).

3. __ The "Small Worlds" axiom for stable equilibria, CORE DP 9007, Université Catholique de Louvain, Louvain-La-Neuve, 1990, Games and Economic Behaviour 16 (1991) 694-753.

4. C. P. Rourke and B. J. Sanderson, Piecewise linear topology, Springer Verlag, Berlin, Heidelberg and New York, 1982.

5. E. H. Spanier, Algebraic topology, McGraw Hill, New York, 1968.

6. R. J. Stern, On topological and piecewise-linear vector fields, Topology, vol. 14, 1975, pp. 257-269.

Center for Operations Research and Econometrics, Universite Catholique de Louvain, B-1348 Louvain-LA-Neuve, Belgium

Department of Economics, SUNY, Stony-Brook, New York 11794 